IJMMS 26:12 (2001) 759-764

PII. S0161171201005798

http://ijmms.hindawi.com

(c) Hindawi Publishing Corp.

\title{
STABLE RINGS GENERATED BY THEIR UNITS
}

\author{
HUANYIN CHEN
}

(Received 12 September 2000)

\begin{abstract}
We introduce the class of rings satisfying $(m, 1)$-stable range and investigate equivalent characterizations of such rings. These give generalizations of the corresponding results by Badawi (1994), Ehrlich (1976), and Fisher and Snider (1976).
\end{abstract}

2000 Mathematics Subject Classification. 19B10, 16E50.

Let $R$ be an associative ring with identity. A ring $R$ is said to have stable range one provided that $a R+b R=R$ implies that $a+b y \in U(R)$ for $y \in R$. It is well known that $M_{R}$ cancels from direct sums if $\operatorname{End}_{R} M$ has stable range one. For further properties of stable range one condition, we refer the reader to [1, 2, 5, 7, 9, 10, 13, 14].

Many authors have studied rings generated by their units (see [3, 4, 7, 8, 10, 12]). It was shown that every unit-regular ring in which 2 is invertible is generated by its unit (see [7, Theorem 5]) and every strongly $\pi$-regular ring in which 2 is invertible is generated by its units (see [8, Theorem 3]). So far one always investigate such rings under stable range one condition.

In this paper, we generalize stable range one condition and introduce rings satisfying $(m, 1)$-stable range so as to investigate rings generated by their units. Also we give generalizations of the corresponding results in [3, 7, 8].

Throughout, rings are associative with identity and modules are right modules. $\mathrm{GL}_{n}(R)$ denotes the general linear group of $R, U(R)$ denotes the set of units of $R$, and that $U_{m}(R)=\left\{x \in R \mid \exists u_{1}, \ldots, u_{m} \in U(R)\right.$ such that $\left.x=u_{1}+\cdots+u_{m}\right\}$. Let $B_{i j}(x)=$ $I_{2}+x e_{i j}(i \neq j, 1 \leq i, j \leq 2),[\alpha, \beta]=\alpha e_{11}+\beta e_{22}$, where $e_{i j}(1 \leq i, j \leq 2)$ are matrix units ( 1 in the $i, j$ position and 0 elsewhere).

DEFINITION 1. The ring $R$ is said to satisfy $(m, 1)$-stable range provided that $a R+$ $b R=R$ implies that $a+b y \in U(R)$ for $y \in U_{m}(R)$.

PROPOSITION 2. The following are equivalent:

(1) The ring $R$ satisfies $(m, 1)$-stable range.

(2) Whenever $a x+b=1$, there exists $y \in U_{m}(R)$ such that $a+b y \in U(R)$.

Proof. $\quad(1) \Rightarrow(2)$. The proof is obvious.

(2) $\Rightarrow(1)$. Given $a R+b R=R$, then $a x+b y=1$ for some $x, y \in R$. So we can find $z \in U_{m}(R)$ such that $a x z+b=u \in U(R)$, and then $a x z u^{-1}+b u^{-1}=1$. Hence we have $w \in U_{m}(R)$ such that $a+b u^{-1} w \in U(R)$. Clearly, $u^{-1} w \in U_{m}(R)$, as desired.

Proposition 3. The following are equivalent:

(1) The ring $R$ satisfies $(m, 1)$-stable range.

(2) The ring $R / J(R)$ satisfies $(m, 1)$-stable range. 
Proof. (1) $\Rightarrow(2)$. Given $\bar{a} \bar{x}+\bar{b}=\overline{1}$ in $R / J(R)$, then $a x+(b+r)=1$ for some $r \in$ $J(R)$. Since $R$ satisfies $(m, 1)$-stable range, we have $y \in U_{m}(R)$ such that $a+(b+r) y \in$ $U(R)$. Therefore $\bar{a}+\bar{b} \bar{y} \in U(R / J(R))$ with $\bar{y} \in U_{m}(R / J(R))$, hence $R / J(R)$ satisfies $(m, 1)$-stable range by Proposition 2 .

(2) $\Rightarrow(1)$. Given $a x+b=1$ in $R$, then $\bar{a} \bar{x}+\bar{b}=\overline{1}$ in $R / J(R)$. So there is $\bar{y} \in U_{m}(R / J(R))$ such that $\bar{a}+\bar{b} \bar{y}=\bar{u} \in U(R / J(R))$. Assume that $y=w_{1}+w_{2}+\cdots+w_{m}$ with all $\overline{w_{i}} \in U(R / J(R))$. Since units lift modulo $J(R)$, we may assume that all $w_{i} \in U(R)$ and $u \in U(R)$, and that $a+b\left(w_{1}+w_{2}+\cdots+w_{m}\right)=u+r$ for some $r \in J(R)$. Obviously, $u+r \in U(R)$ and $w_{1}+w_{2}+\cdots+w_{m} \in U_{m}(R)$. Hence $R$ satisfies $(m, 1)$-stable range, as asserted.

THEOREM 4. Let $R$ be an associative ring with identity, $K$ a set of some elements of $R$. Then the following are equivalent:

(1) Whenever $a x+b=1$, there exists $y \in K$ such that $a+b y \in U(R)$.

(2) Whenever $a x+b=1$, there exists $z \in K$ such that $x+z b \in U(R)$.

Proof. $(1) \Rightarrow(2)$. Since $a x+b=1$, we see that $\left(\begin{array}{cc}a & -b \\ 1 & x\end{array}\right)^{-1}=\left(\begin{array}{cc}x & 1-x a \\ -1 & a\end{array}\right) \in \mathrm{GL}_{2}(R)$. Clearly, $x a+(1-x a)=1$. So there exists $z \in K$ such that $x+(1-x a) z=u \in U(R)$. Hence $\left(\begin{array}{cc}a & -b \\ 1 & x\end{array}\right)^{-1}\left(\begin{array}{cc}1 & 0 \\ z & 1\end{array}\right)=\left(\begin{array}{ll}u & * \\ * & *\end{array}\right) \in \mathrm{GL}_{2}(R)$. Thus we know that $\left(\begin{array}{cc}a & -b \\ 1 & x\end{array}\right)^{-1}=\left(\begin{array}{ll}u & * \\ * & *\end{array}\right)\left(\begin{array}{cc}1 & 0 \\ -z & 1\end{array}\right)$. Therefore $\left(\begin{array}{cc}a & -b \\ 1 & x\end{array}\right)=\left(\begin{array}{ll}1 & 0 \\ z & 1\end{array}\right)\left(\begin{array}{ll}u & * \\ * & *\end{array}\right)^{-1}$. Since there is $w \in U(R)$ such that $\left(\begin{array}{l}u \\ * \\ *\end{array}\right)=$ $\left(\begin{array}{ll}1 & 0 \\ * & 1\end{array}\right)\left(\begin{array}{ll}u & 0 \\ 0 & w\end{array}\right)\left(\begin{array}{ll}1 & * \\ 0 & 1\end{array}\right)$, we have $v=w^{-1} \in U(R)$ such that $\left(\begin{array}{ll}u & * \\ * & *\end{array}\right)^{-1}=\left(\begin{array}{ll}1 & * \\ 0 & 1\end{array}\right)\left(\begin{array}{cc}u^{-1} & 0 \\ 0 & v\end{array}\right)\left(\begin{array}{ll}1 & 0 \\ * & 1\end{array}\right)$. Hence $\left(\begin{array}{cc}a & -b \\ 1 & x\end{array}\right)=\left(\begin{array}{cc}1 & 0 \\ z & 1\end{array}\right)\left(\begin{array}{cc}* & * \\ * & v\end{array}\right), v \in U(R)$. So $\left(\begin{array}{cc}1 & 0 \\ -z & 1\end{array}\right)\left(\begin{array}{cc}a & -b \\ 1 & x\end{array}\right)=\left(\begin{array}{ll}* & * \\ * & v\end{array}\right)$. Thus, we see that $x+z b=v \in U(R)$, as required.

$(2) \Rightarrow(1)$. Applying $(1) \Rightarrow(2)$ to the opposite ring $R^{\text {op }}$, we complete the proof.

Theorem 4 is a general result for symmetry of stable range conditions. As applications, we see that stable range one conditions, unit 1-stable range conditions and rings having many unit-regular elements are symmetric. The following result shows that $(m, 1)$-stable range condition is right-left symmetric.

COROLLARY 5. The following are equivalent:

(1) The ring $R$ satisfies ( $m, 1)$-stable range.

(2) Whenever $a x+b=1$, there exists some $z \in U_{m}(R)$ such that $x+z b \in U(R)$.

(3) Whenever $R a+R b=R$, there exists some $z \in U_{m}(R)$ such that $a+z b \in U(R)$.

Proof. $(1) \Leftrightarrow(2)$. Set $K=U_{m}(R)$. Then the equivalence follows by Theorem 4 .

$(3) \Rightarrow(2)$. The proof is trivial.

(2) $\Rightarrow$ (3). Given $R a+R b=R$, then $x a+y b=1$ for some $x, y \in R$. So we have $s \in U_{m}(R)$ such that $s x a+b=u \in U(R)$, hence $u^{-1} s x a+u^{-1} b=1$. Therefore $a+v u^{-1} b \in U(R)$ for some $v \in U_{m}(R)$, as required.

Proposition 6. The following are equivalent:

(1) The ring $R$ satisfies ( $m, 1)$-stable range.

(2) For any $A \in \mathrm{GL}_{2}(R)$, there exists some $w \in U_{m}(R)$ such that $A=$ $[*, *] B_{21}(w) B_{12}(*) B_{21}(*)$.

(3) For any $A \in \mathrm{GL}_{2}(R)$, there exists some $w \in U_{m}(R)$ such that $A=$ $[*, *] B_{12}(*) B_{21}(*) B_{12}(w)$. 
Proof. $(1) \Rightarrow(2)$. Let $A \in \mathrm{GL}_{2}(R)$, and let $A^{-1}=\left(b_{i j}\right)$. Since $b_{11} R+b_{12} R=R$, we can find some $y \in U_{m}(R)$ such that $b_{11}+b_{12} y=u \in U(R)$. We easily check that $A^{-1}=B_{21}\left(b_{21}+b_{22} u^{-1}\right)\left[u, b_{22}-\left(b_{21}+b_{22} y\right) u^{-1} b_{12}\right] B_{12}\left(u^{-1} b_{12}\right) B_{21}(-y)$. Thus $A=$ $[*, *] B_{21}(w) B_{12}(*) B_{21}(*)$ for some $w \in U_{m}(R)$.

(2) $\Rightarrow(1)$. Given $a x+b=1$ in $R$, then we have $\left(\begin{array}{cc}a & b \\ -1 & x\end{array}\right) \in \mathrm{GL}_{2}(R)$. Thus we have a $w \in U_{m}(R)$ such that $\left(\begin{array}{cc}a & b \\ -1 & x\end{array}\right)^{-1}=[*, *] B_{21}(w) B_{12}(*) B_{21}(*)$. Therefore we see that $\left(\begin{array}{cc}a & b \\ -1 & x\end{array}\right)=[*, *] B_{21}(*) B_{12}(*) B_{21}(-y)$ for some $y \in U_{m}(R)$. Consequently, $a+b y \in$ $U(R)$ with $y \in U_{m}(R)$, as desired.

$(1) \Leftrightarrow(3)$. Applying $(1) \Leftrightarrow(2)$ to the opposite ring $R^{\text {op }}$, we complete the proof by the symmetry of $(m, 1)$-stable range conditions.

Let $R$ be generated by $m$ units. If $R$ has stable range one, then it satisfies $(m, 1)$ stable range. Conversely, we easily check that every ring satisfying $(m, 1)$-stable range is generated by $m+1$ units. Now we show that $(m, 1)$-stable range condition is inherited by matrix rings.

LEMMA 7. The following are equivalent:

(1) The ring $R$ satisfies $(m, 1)$-stable range.

(2) Given $a x+b=1$ in $R$, then there exists $y \in R$ such that $a+b y \in U(R)$ and $1-x y \in U_{m}(R)$.

(3) Given $a x+b=1$ in $R$, then there exists $z \in R$ such that $x+z b \in U(R)$ and $1-z a \in U_{m}(R)$.

Proof. $(1) \Rightarrow(2)$. Given $a x+b=1$ in $R$, then $\left(\begin{array}{cc}a & b \\ -1 & x\end{array}\right) \in \mathrm{GL}_{2}(R)$. In view of Proposition 6, we have a $w \in U_{m}(R)$ such that $\left(\begin{array}{cc}a & b \\ -1 & x\end{array}\right)=[*, *] B_{21}(w) B_{12}(*) B_{21}(*)$. So we can find some $-y \in R$ such that $\left(\begin{array}{cc}a & b \\ -1 & x\end{array}\right)=[*, *] B_{21}(w) B_{12}(*) B_{21}(-y)$. Therefore $a+b y \in U(R)$ and $1-x y=-(-1+x y) \in U_{m}(R)$, as required.

(2) $\Rightarrow(1)$. Given $a x+b=1$ in $R$, then there exists some $y \in R$ such that $a+b y=u \in$ $U(R)$ and $1-x y=v \in U_{m}(R)$. So we know that $\left(\begin{array}{cc}a & b \\ -1 & x\end{array}\right)\left(\begin{array}{ll}1 & 0 \\ y & 1\end{array}\right)=\left(\begin{array}{cc}u & b \\ -v & x\end{array}\right)=$ $[*, *] B_{21}(w) B_{12}(*)$ for some $w \in U_{m}(R)$. Thus $\left(\begin{array}{cc}a & b \\ -1 & x\end{array}\right)=[*, *] B_{21}(w) B_{12}(*) B_{21}(-y)$. So we can find $z \in U_{m}(R)$ such that $\left(\begin{array}{cc}1 & 0 \\ z & 1\end{array}\right)\left(\begin{array}{cc}a & b \\ -1 & x\end{array}\right)=[*, *] B_{12}(*) B_{21}(*)$. Consequently, we show that $x+z b \in U(R)$ for some $z \in U_{m}(R)$. Therefore $R$ satisfies $(m, 1)$-stable range by Corollary 5 .

$(1) \Leftrightarrow(3)$. Applying (1) $\Leftrightarrow(2)$ to the opposite ring $R^{\text {op }}$, we complete the proof.

In [6], the author shows that every matrix ring over a ring satisfying unit 1-stable range also satisfies unit 1-stable range. Now we extend [6, Theorem 2.2] to $(m, 1)$ stable range conditions by a similar route.

THEOREM 8. If $R$ satisfies $(m, 1)$-stable range, then so does $M_{n}(R)$ for any $n \geq 1$.

Proof. Given $B C+D=I_{n}$ in $M_{n}(R)$, then $A=\left(\begin{array}{cc}B & D \\ -I_{n} & C\end{array}\right) \in \mathrm{GL}_{2 n}(R)$. Set $A=\left(\mathbb{A}_{i j}\right)(1 \leq$ $i, j \leq 2)$ with all $\mathbb{A}_{i j}=\left(a_{s t}^{i j}\right) \in M_{n}(R)(1 \leq s, t \leq n)$. Then there exist $x_{1}, \ldots, x_{n}, y_{1}, \ldots$, $y_{n} \in R$ such that $a_{11}^{11} x_{1}+\cdots+a_{1 n}^{11} x_{n}+a_{11}^{12} y_{1}+\cdots+a_{1 n}^{12} y_{n}=1, \ldots, a_{n 1}^{11} x_{1}+\cdots+$ $a_{n n}^{11} x_{n}+a_{n 1}^{12} y_{1}+\cdots+a_{n n}^{12} y_{n}=0, a_{11}^{21} x_{1}+\cdots+a_{1 n}^{21} x_{n}+a_{11}^{22} y_{1}+\cdots+a_{1 n}^{22} y_{n}=0, \ldots$, $a_{n 1}^{21} x_{1}+\cdots+a_{n n}^{21} x_{n}+a_{n 1}^{22} y_{1}+\cdots+a_{n n}^{22} y_{n}=0$. In view of Lemma 7 , there is $z_{1} \in R$ such that $a_{11}^{11}+a_{12}^{11} x_{2} z_{1}+\cdots+a_{1 n}^{11} x_{n} z_{1}+a_{11}^{12} y_{1} z_{1}+\cdots+a_{1 n}^{12} y_{n} z_{1}=u_{1} \in U(R)$ and $1-x_{1} z_{1}=v_{1} \in U_{m}(R)$. So we claim that 


$$
[*, *] A[*, *] B_{21}(*)=\left(\begin{array}{ccccccc}
u_{1} & a_{12}^{11} & \cdots & a_{1 n}^{11} & a_{11}^{12} & \cdots & a_{1 n}^{12} \\
0 & b_{22}^{11} & \cdots & b_{2 n}^{11} & b_{21}^{12} & \cdots & b_{2 n}^{12} \\
\vdots & \vdots & \ddots & \vdots & \vdots & \ddots & \vdots \\
0 & b_{n 2}^{11} & \cdots & b_{n n}^{11} & b_{n 1}^{12} & \cdots & b_{n n}^{12} \\
a_{11}^{21} v_{1} & a_{12}^{21} & \cdots & a_{1 n}^{21} & a_{11}^{22} & \cdots & a_{1 n}^{22} \\
\vdots & \vdots & \ddots & \vdots & \vdots & \ddots & \vdots \\
a_{n 1}^{21} v_{1} & a_{n 2}^{21} & \cdots & a_{n n}^{21} & a_{n 1}^{22} & \cdots & a_{n n}^{22}
\end{array}\right) .
$$

Likewise, we have $u_{2}, u_{3}, \ldots, u_{n} \in U(R)$ and $v_{2}, v_{3}, \ldots, v_{n} \in U_{m}(R)$ such that

$$
[*, *] A[*, *] B_{21}(*)=\left(\begin{array}{cccccccc}
u_{1} & * & * & \cdots & * & a_{11}^{12} & \cdots & a_{1 n}^{12} \\
0 & u_{2} & * & \cdots & * & b_{21}^{12} & \cdots & b_{2 n}^{12} \\
0 & 0 & u_{3} & \cdots & * & c_{31}^{12} & \cdots & c_{3 n}^{12} \\
\vdots & \vdots & \vdots & \ddots & \vdots & \vdots & \ddots & \vdots \\
0 & 0 & 0 & \cdots & u_{n} & d_{n 1}^{12} & \cdots & d_{n n}^{12} \\
a_{11}^{21} v_{1} & a_{12}^{21} v_{2} & a_{13}^{21} v_{3} & \cdots & a_{1 n}^{21} v_{n} & a_{11}^{22} & \cdots & a_{1 n}^{22} \\
\vdots & \vdots & \vdots & \ddots & \vdots & \vdots & \ddots & \vdots \\
a_{n 1}^{21} v_{1} & a_{n 2}^{21} v_{2} & a_{n 3}^{21} v_{3} & \cdots & a_{n n}^{21} v_{n} & a_{n 1}^{22} & \cdots & a_{n n}^{22}
\end{array}\right) .
$$

Similar to the consideration in [6, Theorem 2.2] , we can find some $E \in \mathrm{GL}_{n}(R)$ such that $[*, *] A[*, *] B_{21}(*)=[*, *] B_{21}\left(-E^{-1} \operatorname{diag}\left(v_{1}, \ldots, v_{n}\right)\right) B_{12}(*)$. Consequently, $A=$ $[*, *] B_{21}(W) B_{12}(*) B_{21}(*)$ with $W \in U_{m}\left(M_{n}(R)\right)$. So there is $W^{\prime} \in U_{m}\left(M_{n}(R)\right)$ such that

$$
B_{21}\left(W^{\prime}\right)\left(\begin{array}{cc}
B & D \\
-I_{n} & C
\end{array}\right)=[*, *] B_{12}(*) B_{21}(*), \quad \text { so } C+W^{\prime} D \in \mathrm{GL}_{n}(R) .
$$

It follows from Corollary 5 that $M_{n}(R)$ satisfies $(m, 1)$-stable range.

COROLlary 9. Let $R$ satisfy $(m, 1)$-stable range, then every $n \times n$ matrix over $R$ is the sum of $m+1$ invertible matrices.

Proof. Let $A \in M_{n}(R)$. Since $R$ satisfies $(m, 1)$-stable range, so does $M_{n}(R)$ from Theorem 8. As $A M_{n}(R)+I_{n} M_{n}(R)=M_{n}(R)$, we can find some $U \in U_{m}\left(M_{n}(R)\right)$ such that $A+I_{n} \times U=V \in \mathrm{GL}_{n}(R)$. Thus $A=(-U)+V$, as desired.

Recall that a ring $R$ is said to be an exchange ring if for every right $R$-module $A$ and any two decompositions $A=M^{\prime} \oplus N=\bigoplus_{i \in I} A_{i}$, where $M_{R}^{\prime} \cong R_{R}$ and the index set $I$ is finite, then there exist submodules $A_{i}^{\prime} \subseteq A_{i}$ such that $A=M^{\prime} \oplus\left(\bigoplus_{i \in I} A_{i}^{\prime}\right)$. A ring $R$ is said to be strongly $\pi$-regular provided that for any $x \in R$, there exists a positive integer $n$ such that $x^{n}=x^{n+1} y$ for some $y \in R$.

We note that $R$ satisfies $(m, 1)$-stable range if and only if it has stable range one and for any $x, y \in R$, there exists $w \in U_{m}(R)$ such that $x y+x w+1 \in U(R)$. By an argument 
of M. Henriksen [11], we claim that the ring $R$ has stable range one if and only if the ring $M_{2}(R)$ satisfies $(3,1)$-stable range. For exchange rings, we now derive the following.

LEMMA 10. Let $R$ be an exchange ring with $1 / 2 \in R$. Then the following are equivalent:

(1) The exchange ring $R$ has stable range one.

(2) The exchange ring $R$ satisfies $(7,1)$-stable range.

Proof. $(2) \Rightarrow(1)$. The proof is clear.

(1) $\Rightarrow$ (2). Given $a x+b=1$ in $R$, then $a+b y \in U(R)$ for $y \in R$. Since $R$ is an exchange ring, there exists an idempotent $e \in R$ such that $e=y s$ and $1-e=(1-y) t$. Obviously, $e y$ and $(1-e)(1-y)$ are both regular. Thus $e y=f u,(1-e)(1-y)=g v$ for some $f=f^{2}, g=g^{2} \in R$ and $u, v \in U(R)$. Hence $y=e y-(1-e)(1-y)+1-e=f u-$ $g v+1-e$. As $2 \in U(R)$, we see that $f=2^{-1}+2^{-1}(2 f-1), g=2^{-1}+2^{-1}(2 g-1)$ and $e=2^{-1}+2^{-1}(2 e-1)$. Clearly, $2^{-1}(2 f-1), 2^{-1}(2 g-1), 2^{-1}(2 e-1) \in U(R)$. Therefore $y \in U_{7}(R)$, as required.

THEOREM 11. Let $R$ be a strongly $\pi$-regular ring. If 2 is a nonnilpotent of $R$, then there exists some nonzero idempotent $e \in R$ such that $M_{n}(e R e)$ satisfies $(7,1)$-stable range.

Proof. Since $R$ is a strongly $\pi$-regular ring, there exists $n \geq 1$ such that $2^{n}=e u$ for some $e=e^{2}, u \in U(R)$. Since 2 is a nonnilpotent of $R$, we see that $e \neq 0$. Assume that $u v=1$ for $v \in R$. We easily check that (eue) $($ eve $)=2^{n} e v e=e u v e=e$. Likewise, we have $(e v e)(e u e)=e$. Thus $2 e \in U(e R e)$. On the other hand, we know that $e$ Re is a strongly $\pi$-regular ring. By virtue of [1, Theorem 4 ], $R$ has stable range one. Thus we complete the proof by Theorem 8 and Lemma 10.

Proposition 12. The following are equivalent:

(1) The ring $R$ satisfies $(m, 1)$-stable range.

(2) Whenever $a R+b R=d R$, there exist $y \in U_{m}(R), u \in U(R)$ such that $a+b y=d u$.

(3) Whenever $R a+R b=d R$, there exist $z \in U_{m}(R), u \in U(R)$ such that $a+z b=u d$.

Proof. $(1) \Rightarrow(2)$. Given $a R+b R=d R$, then $(a, b) M_{2}(R)=(d, 0) M_{2}(R)$. Assume that $(d, 0) A=(a, b)$ and $(a, b) B=(d, 0)$. From $A B+\left(I_{2}-A B\right)=I_{2}$, we have $Y \in M_{2}(R)$ such that $A+\left(I_{2}-A B\right) Y=W \in \mathrm{GL}_{2}(R)$. Thus $(a, b)=(d, 0) A=(d, 0)\left(A+\left(I_{2}-A B\right)\right)=$ $(d, 0) W$. Assume that $W=\left(w_{i j}\right)$. Then $w_{11} R+w_{12} R=R$, whence $w_{11}+w_{12} y=u \in$ $U(R)$ for $y \in U_{m}(R)$. Therefore $a+b y=d u$, as desired.

$(2) \Rightarrow(1)$. The proof is trivial.

$(1) \Leftrightarrow(3)$. Applying $(1) \Leftrightarrow(2)$ to the opposite ring $R^{\text {op }}$, we complete the proof by the symmetry of $(m, 1)$-stable range property.

COROLLARY 13. Let $R$ be a ring which is quasi-injective as a right $R$-module. Then the following are equivalent:

(1) The ring $R$ satisfies ( $m, 1)$-stable range.

(2) Whenever $r \cdot \operatorname{ann}(a) \cap r \cdot \operatorname{ann}(b)=r \cdot \operatorname{ann}(d)$, there exists $z \in U_{m}(R)$ such that $r \cdot \operatorname{ann}(a) \cap r \cdot \operatorname{ann}(b)=r \cdot \operatorname{ann}(a+z b)$.

(3) Whenever $l \cdot \operatorname{ann}(a) \cap l \cdot \operatorname{ann}(b)=l \cdot \operatorname{ann}(d)$, there exists $y \in U_{m}(R)$ such that $l \cdot \operatorname{ann}(a) \cap l \cdot \operatorname{ann}(b)=l \cdot \operatorname{ann}(a+b y)$.

Proof. (1) $\Rightarrow(2)$. Suppose $r \cdot \operatorname{ann}(a) \cap r \cdot \operatorname{ann}(b)=r \cdot \operatorname{ann}(d)$. By [5, Proposition 3.4], we claim that $R a+R b=R d$. Using Proposition 12, we can find some $z \in U_{m}(R)$ such 
that $a+z b=d u$ for some $u \in U(R)$. Therefore $r \cdot \operatorname{ann}(a) \cap r \cdot \operatorname{ann}(b)=r \cdot \operatorname{ann}(d)=$ $r \cdot \operatorname{ann}(a+z b)$, as desired.

(2) $\Rightarrow(1)$. Assume that $R a+R b=R$. Then $r \cdot \operatorname{ann}(a) \cap r \cdot \operatorname{ann}(b)=r \cdot \operatorname{ann}(1)$. Thus, we claim that $r \cdot \operatorname{ann}(a) \cap r \cdot \operatorname{ann}(b)=r \cdot \operatorname{ann}(a+z b)$ for a $z \in U_{m}(R)$. Therefore $r \cdot \operatorname{ann}(1)=r \cdot \operatorname{ann}(a+z b)$. By [5, Proposition 3.4], we show that $R=R(a+z b)$, and then $a+z b=u$ is left invertible in $R$. Assume that $v u=1$ for some $v \in R$. From $R v+R(1-u v)=R$, we also have $w \in U_{m}(R)$ such that $v+w(1-u v)=t$ is left invertible in $R$. Clearly, we have $t u=(v+w(1-u v)) u=1$. Hence $t$ is a unit of $R$. Therefore $a+z b=u$ is a unit of $R$, as desired.

$(1) \Leftrightarrow(2)$. By the symmetry of $(m, 1)$-stable range condition, we complete the proof.

ACKNOWLEDGEMENTS. This work was supported by grant no. 19801012 of the National Natural Science Foundation of China, and the Ministry of Education of China. The author would like to thank the referee for many helpful suggestions.

\section{REFERENCES}

[1] P. Ara, Strongly $\pi$-regular rings have stable range one, Proc. Amer. Math. Soc. 124 (1996), no. 11, 3293-3298. MR 97a:16024. Zbl 865.16007.

[2] P. Ara, K. R. Goodearl, K. C. O'Meara, and E. Pardo, Separative cancellation for projective modules over exchange rings, Israel J. Math. 105 (1998), 105-137. MR 99g:16006. Zbl 908.16002.

[3] A. Badawi, On semicommutative $\pi$-regular rings, Comm. Algebra 22 (1994), no. 1, 151157. MR 94j:16015. Zbl 803.16008.

[4] V. P. Camillo and H.-P. Yu, Exchange rings, units and idempotents, Comm. Algebra 22 (1994), no. 12, 4737-4749. MR 95d:16013. Zbl 811.16002.

[5] M. J. Canfell, Completion of diagrams by automorphisms and Bass' first stable range condition, J. Algebra 176 (1995), no. 2, 480-503. MR 97a:16004. Zbl 839.16007.

[6] H. Chen, Units, idempotents, and stable range conditions, Comm. Algebra 29 (2001), no. 2, 703-717. CMP 1841993.

[7] G. Ehrlich, Units and one-sided units in regular rings, Trans. Amer. Math. Soc. 216 (1976), 81-90. MR 52\#8183. Zbl 298.16012.

[8] J. W. Fisher and R. L. Snider, Rings generated by their units, J. Algebra 42 (1976), no. 2, 363-368. MR 54\#7531. Zbl 335.16014.

[9] K. R. Goodearl, von Neumann Regular Rings, Monographs and Studies in Mathematics, vol. 4, Pitman, Massachusetts, 1979. MR 80e:16011. Zbl 411.16007.

[10] D. Handelman, Perspectivity and cancellation in regular rings, J. Algebra 48 (1977), no. 1, 1-16. MR 56\#5642. Zbl 363.16009.

[11] M. Henriksen, Two classes of rings generated by their units, J. Algebra 31 (1974), 182-193. MR 50\#2238. Zbl 285.16009.

[12] R. Raphael, Rings which are generated by their units, J. Algebra 28 (1974), 199-205. MR 49\#7300. Zbl 271.16013.

[13] L. N. Vaserstein, Bass's first stable range condition, J. Pure Appl. Algebra 34 (1984), no. 2-3, 319-330. MR 86c:18009. Zbl 547.16017.

[14] H.-P. Yu, Stable range one for exchange rings, J. Pure Appl. Algebra 98 (1995), no. 1, 105-109. MR 96g:16006. Zbl 837.16009.

Huanyin Chen: Department of Mathematics, Hunan Normal University, Changsha 410006, CHINA

E-mail address: chyzx1@sparc2. hunnu.edu.cn 


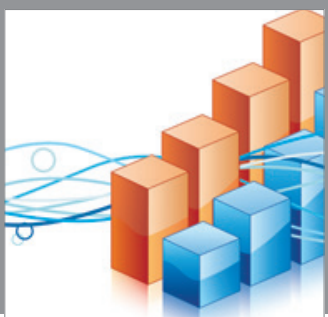

Advances in

Operations Research

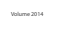

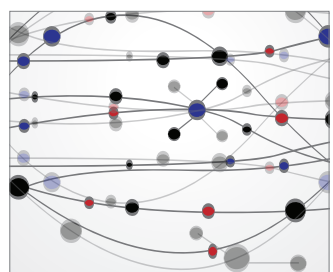

\section{The Scientific} World Journal
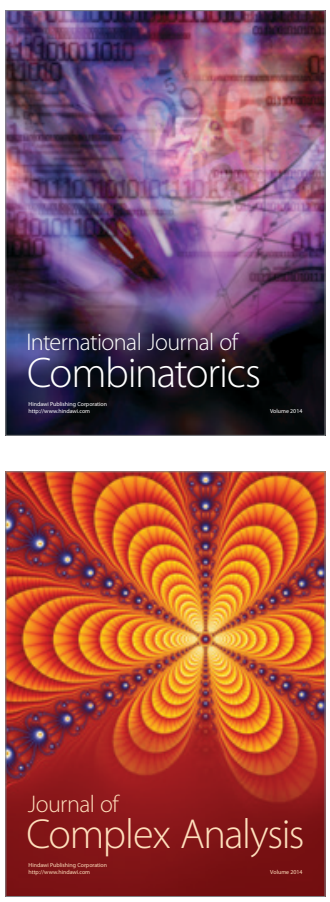

International Journal of

Mathematics and

Mathematical

Sciences
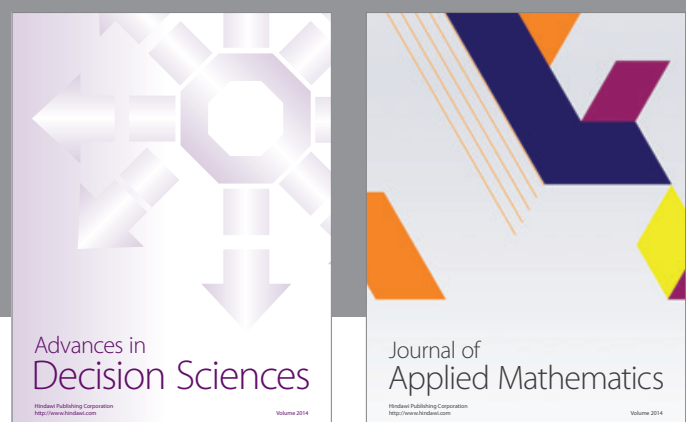

Journal of

Applied Mathematics
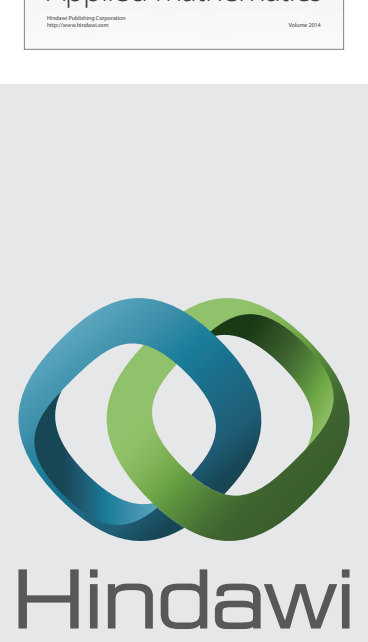

Submit your manuscripts at http://www.hindawi.com
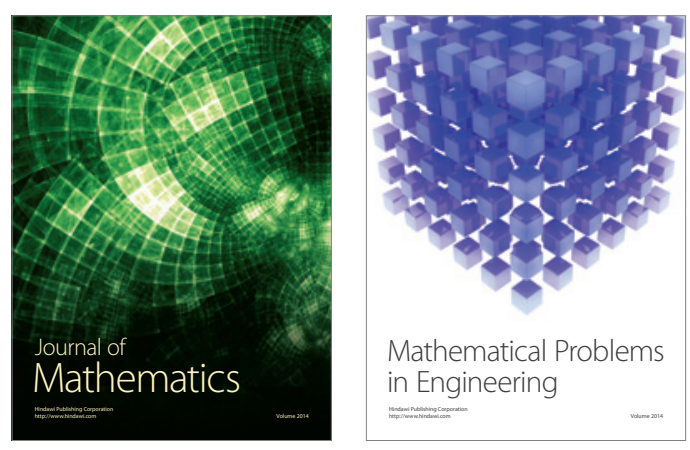

Mathematical Problems in Engineering
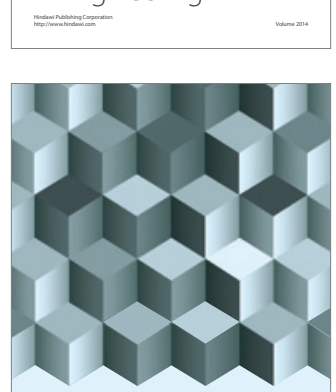

Journal of

Function Spaces
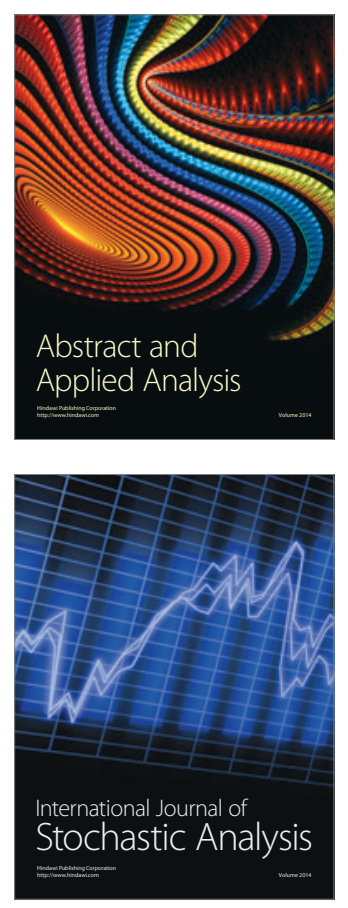

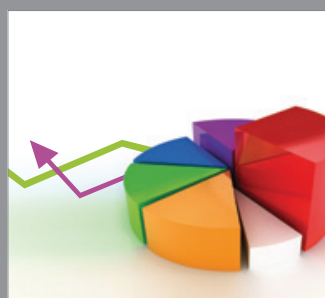

ournal of

Probability and Statistics

Promensencen
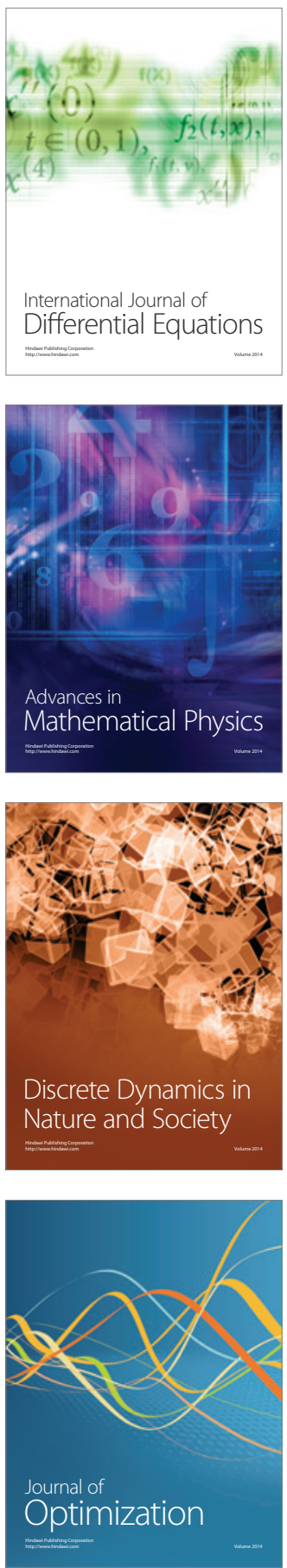